\title{
GLINT: GlucoCEST in neoplastic tumors at 3 T_clinical results of GlucoCEST in gliomas
}

\author{
Benjamin Bender ${ }^{1,6} \cdot$ Kai Herz $^{2,3} \cdot$ Anagha Deshmane $^{2} \cdot$ Vivien Richter $^{1} \cdot$ Ghazaleh Tabatabai $^{4,5,6,7,8}$. \\ Jens Schittenhelm ${ }^{6,9} \cdot$ Marco Skardelly $^{6,10,11} \cdot$ Klaus Scheffler $^{2,3} \cdot$ Ulrike Ernemann $^{1} \cdot$ Mina Kim $^{12} \cdot$ Xavier Golay $^{12}$. \\ Moritz Zaiss ${ }^{2,13} \cdot$ Tobias Lindig $^{1,2}$ (1)
}

Received: 28 June 2021 / Revised: 24 November 2021 / Accepted: 26 November 2021 / Published online: 10 December 2021

(c) The Author(s) 2021

\begin{abstract}
Objective Clinical relevance of dynamic glucose enhanced (DGE) chemical exchange saturation transfer (CEST) imaging has mostly been demonstrated at ultra-high field (UHF) due to low effect size. Results of a cohort study at clinical field strength are shown herein.

Materials and methods Motion and field inhomogeneity corrected T1 $\rho$-based DGE (DGE $\rho$ ) images were acquired before, during and after a D-glucose injection with $6.3 \mathrm{~s}$ temporal resolution to detect accumulation in the brain. Six glioma patients with clear blood-brain barrier (BBB) leakage, two glioma patients with suspected BBB leakage, and three glioma patients without BBB leakage were scanned at $3 \mathrm{~T}$.

Results In high-grade gliomas with BBB leakage, D-glucose uptake could be detected in the gadolinium (Gd) enhancing region as well as in the tumor necrosis with a maximum increase of $\triangle \mathrm{DGE} \rho$ around $0.25 \%$, whereas unaffected white matter did not show any significant DGE $\rho$ increase. Glioma patients without Gd enhancement showed no detectable DGE $\rho$ effect within the tumor.

Conclusion First application of DGE $\rho$ in a patient cohort shows an association between BBB leakage and DGE signal irrespective of the tumor grade. This indicates that glucoCEST corresponds more to the disruptions of BBB with Gd uptake than to the molecular tumor profile or tumor grading.
\end{abstract}

Keywords glucoCEST $\cdot$ Dynamic glucose enhancement - Glioblastoma $\cdot$ Chemical exchange saturation transfer $\cdot$ CEST

Tobias Lindig

tobias.lindig@med.uni-tuebingen.de

1 Department of Diagnostic and Interventional Neuroradiology, University Hospital Tübingen, Hoppe-Seyler-Str. 3, 72076 Tübingen, Germany

2 Magnetic Resonance Center, Max Planck Institute for Biological Cybernetics, Tübingen, Germany

3 Department of Biomedical Magnetic Resonance, University Hospital Tübingen, Tübingen, Germany

4 Department of Neurology and Interdisciplinary Neuro-Oncology, University Hospital Tübingen, Tübingen, Germany

5 Hertie Institute for Clinical Brain Research, Eberhard Karls University Tübingen, Tübingen, Germany

6 Center for Neuro-Oncology, Comprehensive Cancer Center Tübingen-Stuttgart, University Hospital Tübingen, Eberhard Karls University Tübingen, Tübingen, Germany
7 Cluster of Excellence iFIT (EXC 2180) "Image Guided and Functionally Instructed Tumor Therapies", Eberhard Karls University Tübingen, Tübingen, Germany

8 German Consortium for Translational Cancer Research (DKTK), Partner Site Tübingen, German Cancer Research Center (DKFZ), Tübingen, Germany

9 Department of Neuropathology, University Hospital Tübingen, Tübingen, Germany

10 Department of Neurosurgery, University Hospital Tübingen, Tübingen, Germany

11 Department of Neurosurgery, Klinikum am Steinenberg, Reutlingen, Germany

12 Institute of Neurology, University College London, London, UK

13 Department of Neuroradiology, University Hospital Erlangen, Friedrich-Alexander University Erlangen-Nürnberg (FAU), Erlangen, Germany 


\section{Introduction}

Chemical exchange saturation transfer (CEST) has become a promising tool, for the depiction of micro-environmental and metabolic information within the human brain by utilizing the chemical exchange between protons of protein/ metabolites and the abundant water proton pool. Glucose exhibits a hydroxyl CEST effect and was identified early as a potential natural contrast agent to image D-glucose uptake and metabolism in tumors [1,2]. After the first successful application in the animal brain tumor model [3], the method was quickly also applied in humans at ultrahigh field strengths [4-9]. Due to the current problems of scanning patients at ultra-high field (UHF) strengths, and particularly its lack of availability, a translation to a clinical field strength of 3 Tesla ( $3 \mathrm{~T}$ ) is needed to increase the method's reach and ensure its broader clinical evaluation. At ultra-high field strengths of $7 \mathrm{~T}$ and above the CEST effect is stronger than at $3 \mathrm{~T} \mathrm{[10],} \mathrm{and} \mathrm{the} \mathrm{frequency}$ separation between resonances of the exchangeable groups and the water peak is larger, leading to an improvement in signal detection. While subject motion can cause a pseudoCEST-effect [11], nonetheless, successful implementations of chemical exchange saturation transfer sequences at $3 \mathrm{~T}$ have been recently reported $[12,13]$.

The exact underlying mechanism for the glucoCEST contrast is still under debate. Potential influencing factors are the perfusion, the permeability of the blood-brain barrier, the uptake kinetics of glucose and the local (tumor) metabolism which leads through the Warburg effect to a decrease of $\mathrm{pH}$ in the interstitial space [2], but the effect and interaction of these factors in-vivo are not clear. In this prospective study, we use a previously optimized 3D chemical exchange saturation spinlock (CESL) sequence to measure T1 $\rho$-based dynamic D-glucose enhanced (DGE $\rho$ ) signal in a cohort of patients with brain tumors. We examined the effects associated with blood-brain barrier breakdown and molecular tumor type and grade.

\section{Materials and methods}

\section{Patients}

This prospective study was conducted between 2018 and 2020 after prior approval by the local institutional review board (Ethics committee of the Medical Faculty at the Eberhard-Karls University of Tübingen and University Hospital Tübingen). All patients gave their written informed consent prior to the measurements. A total of 11 patients with suspected primary brain tumors were included in the study. Besides the DGE $\rho$-weighted CESL sequence, routine MRI data were available for all patients, which consisted of T1-w, T2-w and fluid-attenuated inversion recovery weighted (FLAIR-w) images without contrast agent and a post-T1-w contrast scan. Blood-brainbarrier breakdown was assessed by the presence of gadolinium contrast agent uptake within the tumor. All but one patient underwent concurrent resection or biopsy. Demographics and clinical information of all patients are summarized in Table 1. The first three patients have been included in a previous preliminary evaluation [12].

\section{MRI measurements}

All imaging was performed at a clinical $3 \mathrm{~T}$ scanner (Prisma; Siemens Healthcare, Erlangen, Germany) with a 64-channel Rx Head Coil for optimal SNR. We applied a previously 3 T-optimized DGE-weighted CESL gradient echo (GRE) 3D snapshot sequence [12]. In short, the protocol consists of repeated blocks with $4 \mathrm{~s}$ of relaxation, a saturation phase (adiabatic prepared SL pulse of $4 \mu \mathrm{T}$ and $120 \mathrm{~ms}$ locking time) [12] and snapshot GRE readout (Deshmane et al. 2019). Images were acquired with a $2 \times 2 \mathrm{~mm}^{2}$ in-plane resolution (FoV $180 \times 220 \mathrm{~mm}$, bandwidth $700 \mathrm{~Hz} / \mathrm{px}$ ), with 12 slices ( $5 \mathrm{~mm}$ slice thickness), flip angle of $6^{\circ}$ and parallel imaging acceleration factor 2 in phase encoding direction with GRAPPA reconstruction performed offline. The dynamic measurement included an unsaturated M0 scan at the beginning, followed by 32 measurements at each of five different frequency offsets $(-300,0.6,0.9,1.2$, and $1.5 \mathrm{ppm})$. All frequency offsets were measured after each other, resulting in 32 groups of 5 measurements each and a total of 161 measurements, including an M0 dummy scan at the beginning with a total scan time of $16: 45 \mathrm{~min}$, a temporal resolution of $6.3 \mathrm{~s}$ per scan and $31 \mathrm{~s}$ per group of offset measurements. If the measurement was part of a clinical scan, a contrast agent was applied after the DGE-weighted sequences.

\section{Glucose injection protocol}

Prior to D-glucose infusions blood levels of potassium and sodium were checked and a baseline glucose level was measured to verify the safety of D-glucose injection $(<160 \mathrm{mg} /$ dl). Patients were asked to fasten for $12 \mathrm{~h}$, but some patients did not adhere to this request. A $20 \mathrm{G}$ or $21 \mathrm{G}$ peripheral venous catheter (PVC) was placed in the antecubital fossa and correct placement was tested with a $10 \mathrm{ml} 0.9 \%$ saline flush. The patient was placed within the scanner, and a flushed $2 \mathrm{~m}$ perfusion line was attached to the PVC. After a baseline measurement of $3 \mathrm{~min}$ a D-glucose bolus of $1 \mathrm{ml}$ clinical D20 glucose injection solution (20 g dextrose in $100 \mathrm{ml}$ ) per kilogram body weight (e.g. $70 \mathrm{ml}$, equal to $14 \mathrm{~g}$ 
Table 1 Demographics, tumor location, tumor type, molecular tumor profile and progression-free survival for all patients

\begin{tabular}{|c|c|c|c|c|c|c|}
\hline ID & Gender, age & Tumor location & Tumor type & Molecular status & BBB break down & $\begin{array}{l}\text { Recurrency/pro- } \\
\text { gressive disease } \\
\text { (months) }\end{array}$ \\
\hline 1 & M, 70 & $\mathrm{R}$ parietal & $\mathrm{GBM}^{\circ} \mathrm{IV}$ & $\begin{array}{l}\text { IDH1/2 WT, ATRX reten- } \\
\text { tion, MGMT non-meth- } \\
\text { ylated }\end{array}$ & Yes & 21 \\
\hline 2 & $\mathrm{~F}, 61$ & $\mathrm{~L}$ parieto-temporal & $\mathrm{GBM}^{\circ} \mathrm{IV}$ & $\begin{array}{l}\text { IDH1/2 WT, ATRX reten- } \\
\text { tion, MGMT non-meth- } \\
\text { ylated }\end{array}$ & No & 3 \\
\hline 3 & $\mathrm{~F}, 54$ & R temporal & Giant cell GBM ${ }^{\circ} \mathrm{IV}$ & $\begin{array}{l}\text { IDH1/2 WT, ATRX reten- } \\
\text { tion, MGMT intermediate } \\
\text { methylated }\end{array}$ & Yes & \\
\hline 4 & $\mathrm{~F}, 46$ & R Temporo-insular & Diffuse astro-cytoma ${ }^{\circ} \mathrm{II}$ & $\begin{array}{l}\text { IDH1 pos, } 1 \mathrm{p} / 19 \mathrm{q} \text { no } \mathrm{LoH} \text {, } \\
\text { ATRX retention }\end{array}$ & No & \\
\hline 5 & $\mathrm{~F}, 29$ & $\mathrm{~L}$ frontal & Diffuse astro-cytoma ${ }^{\circ} \mathrm{II}$ & $\begin{array}{l}\text { IDH1 pos, 1p/19q no LoH, } \\
\text { ATRX loss }\end{array}$ & (Yes) & \\
\hline 6 & M, 70 & R temporo-insular-frontal & Anaplastic astro-cytoma ${ }^{\circ} \mathrm{III}$ & $\begin{array}{l}\text { IDH1/2 WT, ATRX reten- } \\
\text { tion, MGMT methylated }\end{array}$ & No & 11 \\
\hline 7 & $\mathrm{~F}, 63$ & $\mathrm{~L}$ insular & $\mathrm{GBM}^{\circ} \mathrm{IV}$ & $\begin{array}{l}\text { IDH1/2 WT, ATRX reten- } \\
\text { tion, MGMT non-meth- } \\
\text { ylated }\end{array}$ & Yes & \\
\hline 8 & F, 52 & R occipital & $\mathrm{GBM}^{\circ} \mathrm{IV}$ & $\begin{array}{l}\text { IDH1/2 WT, ATRX reten- } \\
\text { tion, MGMT non-meth- } \\
\text { ylated }\end{array}$ & Yes & 15 \\
\hline 9 & F, 58 & $\mathrm{R}$ parieto-insular & $\mathrm{GBM}^{\circ} \mathrm{IV}$ & $\begin{array}{l}\text { IDH1/2 WT, ATRX reten- } \\
\text { tion, MGMT non-meth- } \\
\text { ylated }\end{array}$ & Yes & 7 \\
\hline 10 & M, 69 & $\mathrm{~L}$ parietal & $\mathrm{GBM}^{\circ} \mathrm{IV}$ & $\begin{array}{l}\text { IDH1/2 WT, ATRX reten- } \\
\text { tion, MGMT methylated }\end{array}$ & Yes & 1 \\
\hline 11 & M, 43 & $\mathrm{~L}$ fronto-insular & LGG & N/A & (Yes) & \\
\hline
\end{tabular}

$B B B$ blood-brain barrier (= contrast enhancement), $F$ female, $G B M$ glioblastoma multiforme, $I D H$ isocitrate dehydrogenase, $L$ left, $L G G$ lowgrade glioma, $L o H$ loss of heterozygosity, $M$ male, MGMT $O$-6-methylguanine-DNA-methyltransferase, $R$ right, $W T$ wild type, (Yes) faint contrast enhancement

dextrose for a patient with $70 \mathrm{~kg}$ body weight) was injected manually over a time period of 2-2.5 min and the perfusion line was flushed afterwards with $0.9 \%$ saline. At the end of the MR measurement blood glucose levels were measured again for safety reasons to rule out induced relevant hyperglycemia or reactive hypoglycemia.

\section{Postprocessing}

Postprocessing was performed as in Ref. [12] and consisted of a retrospective rigid body motion correction using elastix [14], a dynamic B0 correction [15] using the phase information of the GRE readout and a Z-image calculation. Z-images were normalized dynamically by the corresponding measurement at $-300 \mathrm{ppm}$. For the B0 correction, $Z$-values off a subsequent group of offsets $(0.6,0.9,1.2$ and 1.5$)$ were linearly interpolated voxel-wise. Afterwards, $\triangle \mathrm{DGE} \rho$ difference maps to baseline were calculated at each time-point and offset, using the mean of the first six images per offset as a baseline and the resulting DGE $\rho$ signal was filtered in time dimension by a box filter to get a more stable signal [12]. For visualization, the $\triangle \mathrm{DGE} \rho$ maps were filtered spatially, again using a box filter.

\section{Evaluation}

All resulting images were rated visually for the presence of obvious motion artifacts. Patients with extreme motion were excluded from the following analysis. Presence of signal change after D-glucose injection, the start of signal change $t_{\text {(start) }}$ after injection, time to maximum $t_{(\max )}$ after injection, and maximum signal change $\Delta \mathrm{DGE} \rho_{(\max )}$ were evaluated for all patients. To reduce noise, mean $\triangle \mathrm{DGE} \rho$ values from 8 to 10 min post-injection were calculated as well. Spatial correlation between contrast agent uptake and DGE $\rho$ signal was evaluated visually by two neuroradiologists (BB, TL) in a consensus approach.

First and the last slice of the image stack were discarded, after which regions of interest (ROIs) were drawn manually on at least three slices that cover the tumor in each patient 
a
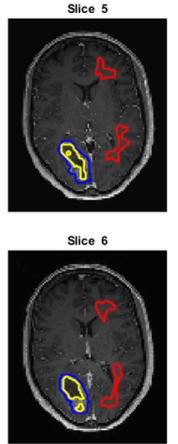

Slice 7

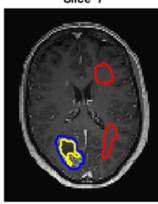

b

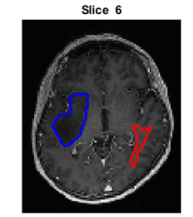

slice 7

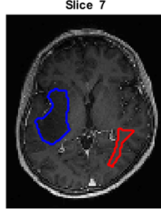

Slice 8

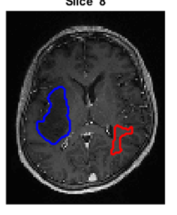

C
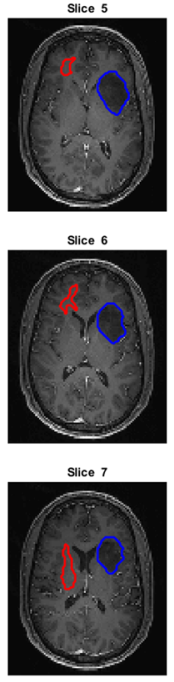
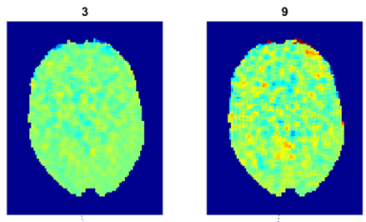

Injection Start Injection Stop

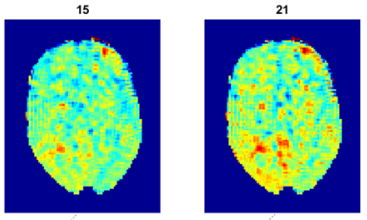

DGE at slices 5-7 at $0.6 \mathrm{ppm}$
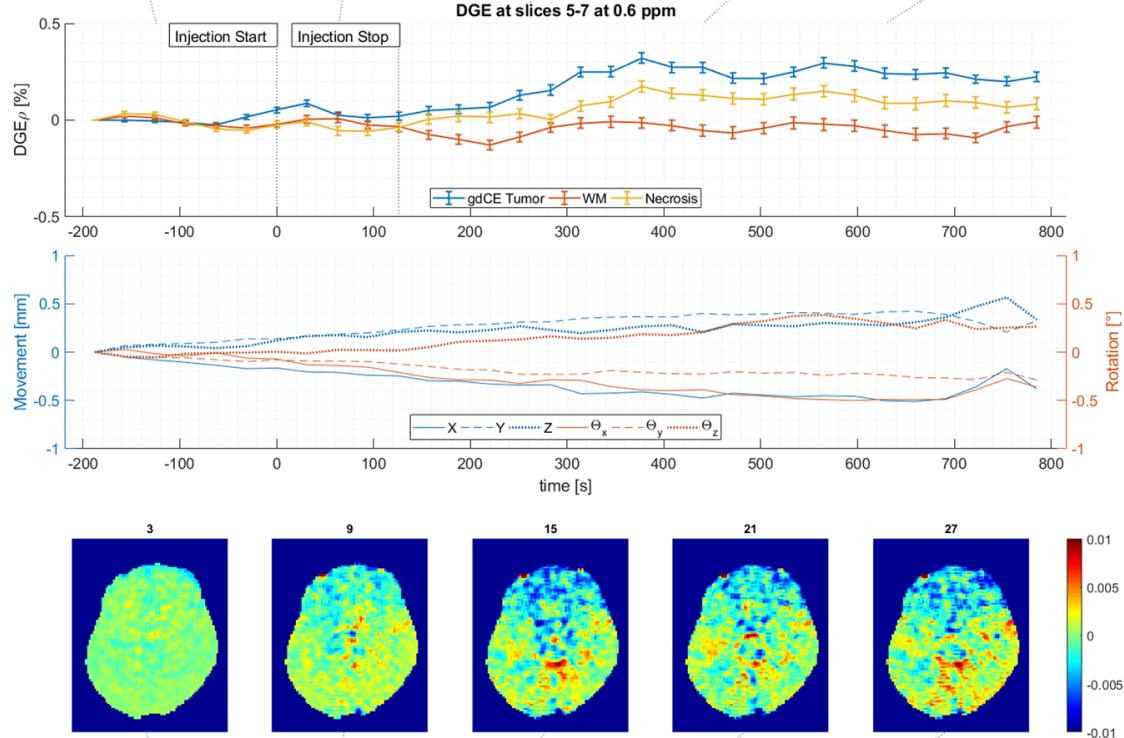

Injection Start
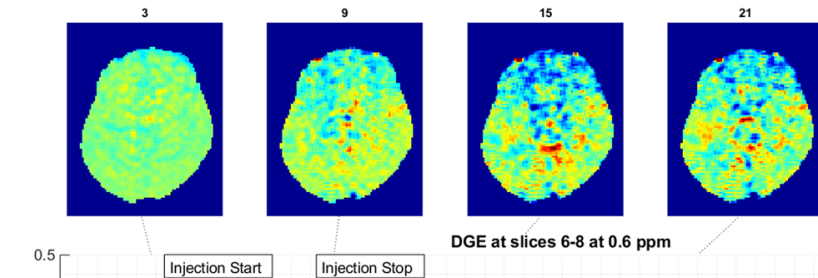

DGE at slices 6-8 at $0.6 \mathrm{ppm}$
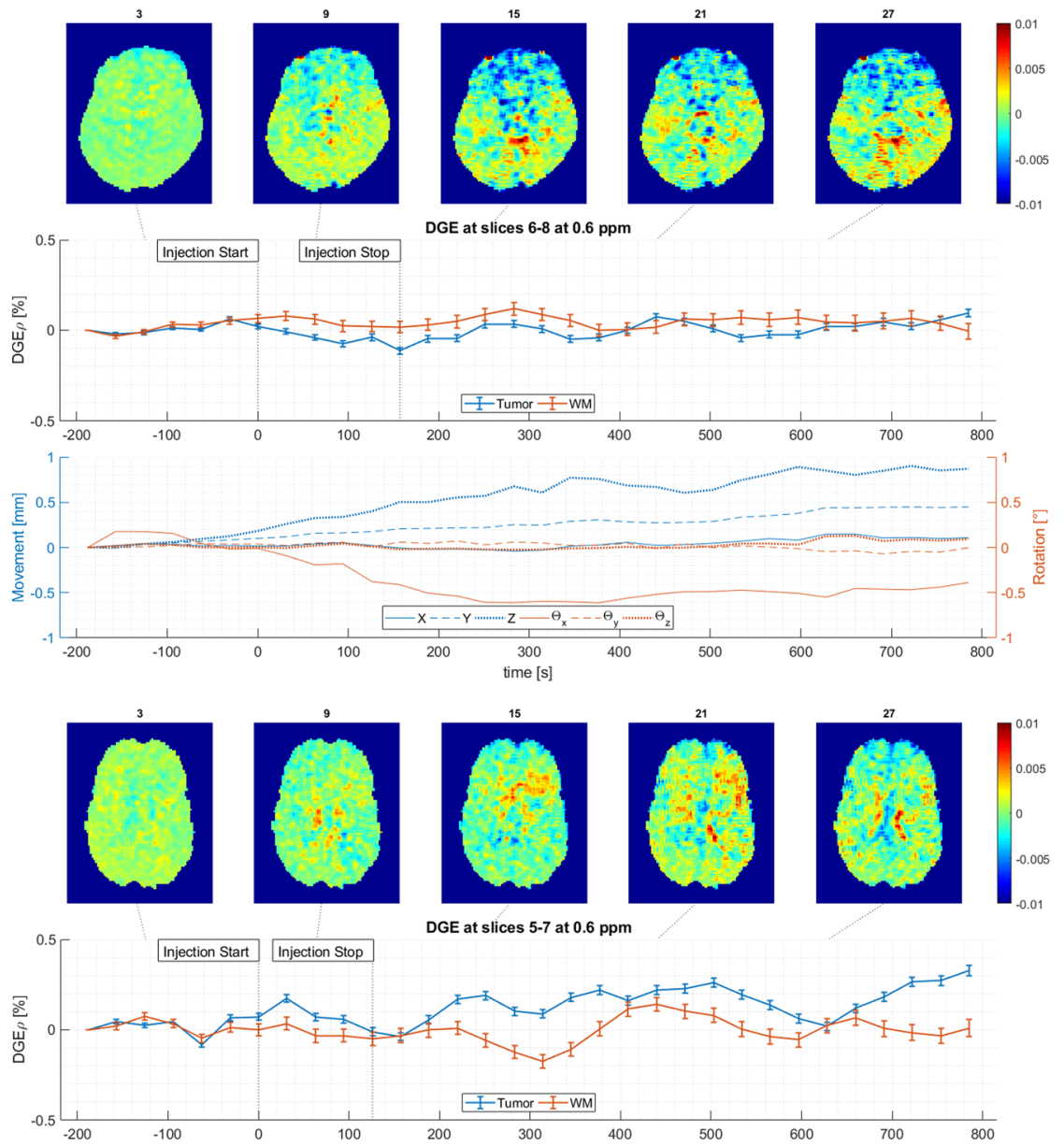

DGE at slices 5-7 at $0.6 \mathrm{ppm}$

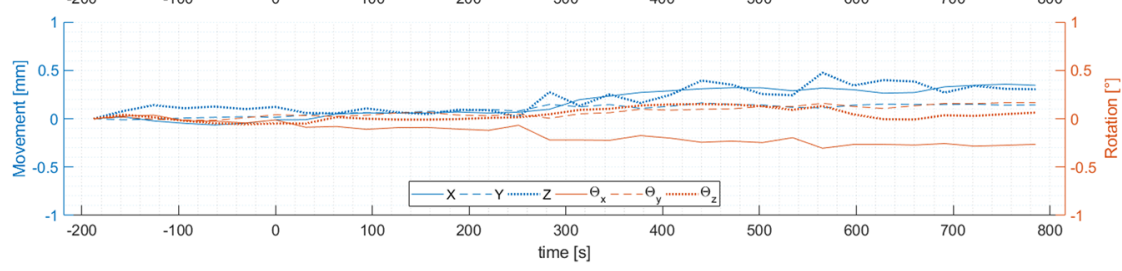


4Fig. $1 \Delta$ DGE $\rho$ images, signal intensity profiles of the regions of interest and motion estimates for three typical examples are displayed, in a patient with clear contrast enhancement (a, patient no. 8), no contrast agent uptake (b, patient no. 4) and a faint contrast agent uptake (c, patient no. 11) of the tumor

by a neuroradiologist (TL). If a clear contrast enhancement of the tumor was seen, contrast-enhancing areas (CE-ROI) and (if present) central necrosis (necrosis-ROI) were identified separately. If no or only faint contrast enhancement was visible, the whole T2-hyperintense tumor (FLAIR-ROI) was chosen as a single ROI, excluding obvious regions of edema. In all patients, an additional ROI in normal-appearing white matter was drawn on the same slices (WM-ROI).

Statistical evaluation was conducted with IBM SPSS Statistics (Version 24.0). A statistical significance was expected for any $p<0.05$ (corrected for multiple comparisons).

\section{Results}

Range and dynamics of motion differed between patients. Patients (no. 1, 7 and 9) with sudden shifts in the position showed more obvious motion artifacts than a constant small shift over time. In patient no. 1 movement was minimal until the late scans (around 10 min after D-glucose injection) where a sudden move was identified; therefore, evaluation was possible until this point of time. Patient no. 7 and 9 were excluded from statistical evaluation due to severe motion artifacts. No increase in DGE $\rho$ signal was seen in patients with no signs of a blood-brain barrier breakdown (no. 2, 4, and 6). In none of these patients was a pseudoCEST contrast due to movement visible. Patients with a strong enhancement and necrosis (no. 1, 3, 8, 9 and 10), and also two patients (no. 5 and 11) with a faint enhancement showed a DGE $\rho$ signal increase starting approximately 4 min after injection with a maximum increase of $\triangle \mathrm{DGE} \rho$ between 0.2 and $0.4 \%$ after approximately $9 \mathrm{~min}$, whereas tumor-unaffected white matter regions did not show any significant DGE $\rho$ increase (see Figs. 1, 2).

The distribution of the mean $\triangle \mathrm{DGE} \rho$ values at $8-10 \mathrm{~min}$ post injection for each group based on all voxels within the ROIs showed a normal distribution (see Supplemental Fig. 1). The effect did not differ between the offsets (see Supplemental Fig. 1), due to the broad DGE $\rho$ effect at this frequency range. We conducted a one-way ANOVA to assess the effect of tissue type (defined by ROIs) on mean $\Delta$ DGE $\rho$ at 8-10 min post injection: WM-ROI $(N=9, M=0.00$, $\mathrm{SD}=0.46)$, FLAIR-ROI $(N=5, M=0.12, \mathrm{SD}=0.18)$, CEROI $(N=5, M=0.19, \mathrm{SD}=0.09)$ and necrosis-ROI $(N=4$, $M=0.20, \mathrm{SD}=0.11)$. Leven's test showed a violation of the assumption of homogeneity ( $p=0.026)$. The mean $\Delta$ DGE $\rho$ at $8-10$ min post injection differed significantly between
ROIs, Welch's $F(3,6.84)=8.16, p=0.012$. Games-Howell post-hoc analysis revealed only a significant difference ( $p=0.028$ ) between WM-ROI and CE-ROI, with an increase in mean $\triangle \mathrm{DGE} \rho$ at $8-10$ min post injection in the CE-ROI $(0.19,95 \%$ CI [0.04-0.34], but not for WM-ROI vs necrosisROI $(p=0.111 ; 0.20,95 \%$ CI $[-0.07$ to 0.41$])$ or FLAIRROI ( $p=0.570 ; 0.1195 \%$ CI [ -0.21 to 0.44$])$.

\section{Discussion}

After the exclusion of two patients due to movement artifacts, a DGE $\rho$ effect could be shown at $3 \mathrm{~T}$ for $6 / 9$ patients with a contrast-enhancing tumor, for which a mean signal increase at $8-10 \mathrm{~min}$ post injection was around $0.19 \%(95 \%$ CI $0.04-0.34 \%$ ). This effect was smaller than a previous report at $7 \mathrm{~T}$ [5], which is in line with theoretical simulations [10], but the time curves nicely match previous published $3 \mathrm{D}$ results at $7 \mathrm{~T}$ that also included motion correction [6]. The measured effect was somewhat smaller than the results in three recent patients measured at $3 \mathrm{~T}$ with a mean signal increase at 2-7 $\mathrm{min}$ post injection of $0.52-0.97 \%$ [13], but the patients characteristics were not comparable. In addition, the dynamic B0 correction and normalization performed herein can also limit overestimations of effects [11, 12]. All patients within our study were treatment-naive, thus the findings are probably based on tumor-induced changes to perfusion, blood-brain barrier leakage, change in local $\mathrm{pH}$ and/or glucose metabolism. In glioblastomas (grade IV) cell densities and BBB leakage is expected to be higher, as in grade II and III tumors, where necrosis is absent. In the recent publication by $\mathrm{Xu}$ et al. post-treatment patients (surgery and probably radio-chemotherapy) were included. In such patients, the effect of therapy on the blood-brain barrier, local necrosis and inflammation can have significant effects on the local signal evolution. An interpretation and comparison of the results are, therefore, difficult.

From the different tumors and contrast agent uptake in our cohort, some careful hypothesis can be generated. It seems that the breakdown of the blood-brain barrier is the most important mediator of the DGE $\rho$ signal in-vivo. Perfusion changes often correlate with contrast enhancement in routine glioma imaging, although both imaging findings are based on different pathophysiologic changes, and thus local perfusion increase should be considered as one of the main drivers for the increase in DGE $\rho$ seen in our patient cohort. The kinetics seen in the DGE $\rho$ signal within the contrast-enhancing ROI do not show a typical perfusion related pattern, with a slow but steady increase over a longer period of time (Fig. 1a), which makes a large perfusion effect unlikely. Also, no DGE $\rho$ signal increase was detectable in histologically proven high-grade glioma with neither contrast enhancement nor signs of necrosis, but 


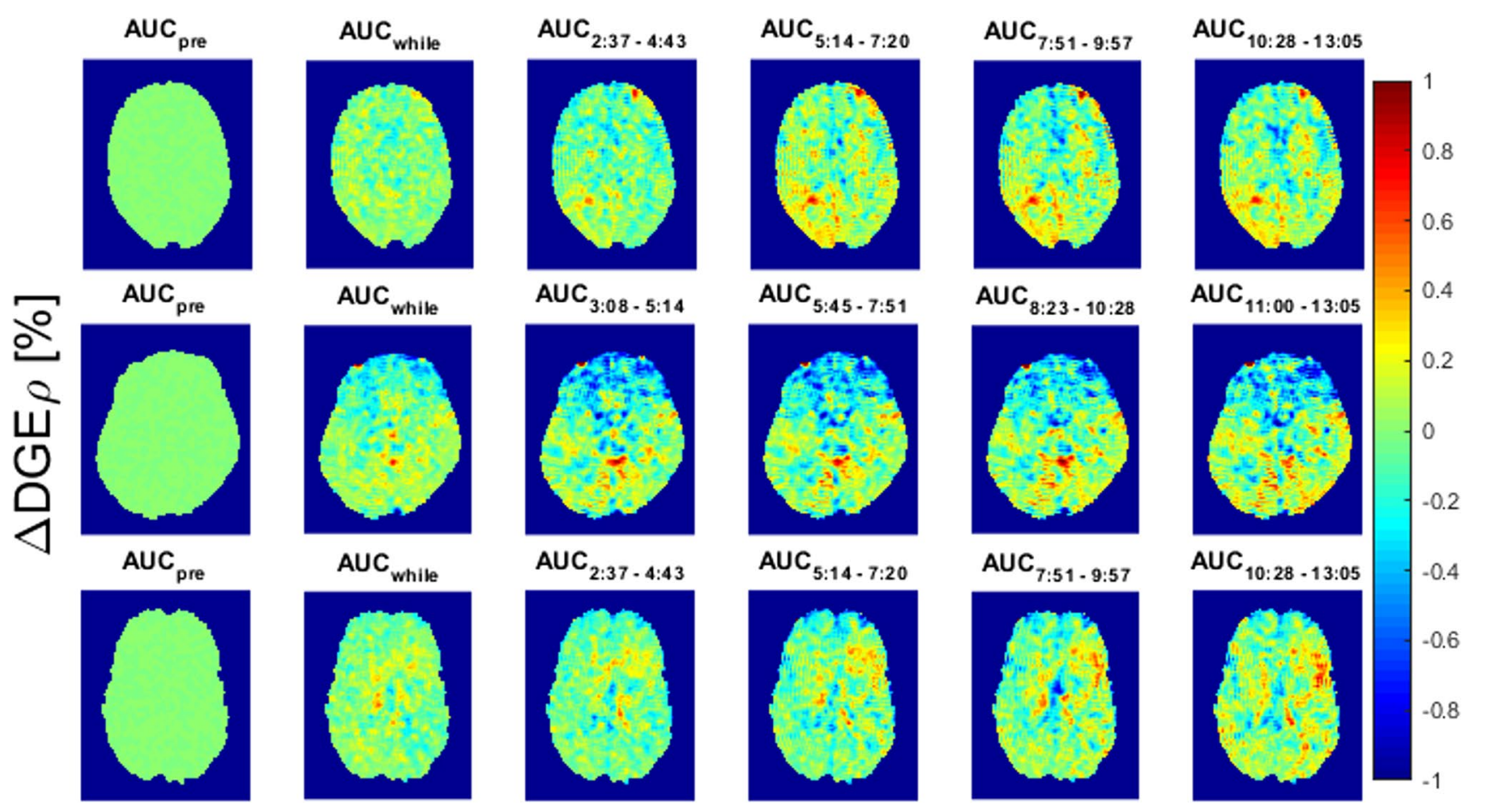

Fig. $2 \triangle$ DGEp AUC maps for patients 8 (1st row, slice 6). 4 (2nd row, slice 7) and 11 (3rd row, slice 6). The maps show the AUC during different timeframes: mean $\triangle \mathrm{DGE} \rho$ values before injection (1st column), during injection (2nd column) and after the injection (3rd-5th column). The time in the subscript indicates the timeframe after the beginning of the injection. Supplemental Fig. 1: $\triangle D G E \rho$

at 8-10 min post-injection for each measured offset: each box plot consists of all voxels within the respective ROIs. ROIs of contrastenhancing tumor, of tumor necrosis, of T2-hyperintense tumor without contrast enhancement, and in normal-appearing white matter were analyzed. Similar effects were measured at all offsets

with increased proliferation rate and increased vascularity (no. 2). On the other hand, one patient with a proven diffuse astrocytoma (no necrosis, no increased vascularity, no. 5 ) and another with a typical imaging finding of a diffuse astrocytoma (no. 11), showed a positive DGE $\rho$ signal and a faint contrast enhancement, although necrosis should be absent and cell density lower than in grade IV tumors. From the results of the individual ROIs (see Table 2) the increase seen in FLAIR-ROIs was only based on the two patients with a faint contrast enhancement (see Table 3), while the other three patients showed similar values as for WM-ROI. The spatial effect was not strictly aligned to the contrast enhancement in all patients, which suggest a potential additional effect, like $\mathrm{pH}$ which is altered in many gliomas $[16,17]$. Lower $\mathrm{pH}$, as expected from extracellular lactate, leads to a lower hydroxyl exchange rate and thus better detectability via DGE [2], especially at lower clinical field strength.

Motion artifacts are the main limitation at $3 \mathrm{~T}$ for DGE imaging, as the artificial effects on imaging can be much higher than the expected physiological changes. As artifacts arise mainly at the borders of tissues, such effects can spatially overlap with contrast-enhancing tumor and mimic physiologic findings [11]. Minimization of movement has therefore been identified as one of the most important tasks

Table 2 Mean $\triangle \mathrm{DGE}$ at $8-10 \mathrm{~min}$ post injection within the individual ROI

\begin{tabular}{lcclc}
\hline Pat no & $\begin{array}{l}\Delta \text { DGE CE } \\
(\%)\end{array}$ & $\begin{array}{l}\Delta \text { DGE } \\
\text { necrosis } \\
(\%)\end{array}$ & $\begin{array}{l}\Delta \text { DGE FLAIR } \\
(\%)\end{array}$ & $\Delta$ DGE WM $(\%)$ \\
\hline 1 & 0.25 & 0.16 & & 0.05 \\
2 & & & -0.02 & 0.00 \\
3 & 0.20 & 0.14 & & -0.01 \\
4 & & & -0.01 & 0.06 \\
5 & & & 0.41 & -0.06 \\
6 & 0.04 & & 0.03 & -0.05 \\
7 & -0.04 & 0.01 & & 0.01 \\
8 & 0.25 & 0.13 & & -0.03 \\
9 & -0.22 & -0.08 & & 0.05 \\
10 & 0.23 & 0.37 & & 0.06 \\
11 & & & 0.18 & 0.02 \\
\hline
\end{tabular}

ROIs of contrast-enhancing tumor, of tumor necrosis, of T2-hyperintense tumor without or faint contrast enhancement, and in normalappearing white matter were analyzed. Patient no. 7 and 9 were not included in the statistical analysis (marked in italic) 
Table 3 Start of visible increase in $\triangle$ DGE signal after glucose injection, time to estimated maximum and maximum values in CE-ROI, maximum motion, result of visual inspection for contrast agent uptake $(\mathrm{o}=$ no, $(+)=$ faint,$+=$ clearly defined $)$ and blood glucose levels

\begin{tabular}{llllllll}
\hline ID & $t_{\text {(start) }}$ in $\mathrm{s}$ & $t_{(\max )}$ in s & $\begin{array}{l}\Delta \mathrm{DGE}_{(\max )} \\
\mathrm{CE} \%\end{array}$ & Max. motion & CE & $\begin{array}{l}\text { Glucose } \\
\text { start MR }\end{array}$ & $\begin{array}{l}\text { Glucose } \\
\text { end MR }\end{array}$ \\
\hline 1 & 15 & 288 & 0.32 & $1.5 \mathrm{~mm} / 0.7^{\circ}$ & ++ & 111 & 158 \\
2 & N/A & N/A & N/A & $0.3 \mathrm{~mm} / 0.4^{\circ}$ & $\mathrm{O}$ & 93 & 134 \\
3 & 15 & 288 & 0.3 & $0.5 \mathrm{~mm} / 0.7^{\circ}$ & ++ & 154 & 201 \\
4 & N/A & N/A & N/A & $0.9 \mathrm{~mm} / 0.5^{\circ}$ & $\mathrm{O}$ & 94 & 140 \\
5 & 15 & 416 & 0.51 & $0.5 \mathrm{~mm} / 0.6^{\circ}$ & $(+)$ & 83 & 124 \\
6 & N/A & N/A & N/A & $0.3 \mathrm{~mm} / 0.8^{\circ}$ & $\mathrm{O}$ & 103 & $98^{\mathrm{a}}$ \\
7 & N/A & N/A & N/A & $0.7 \mathrm{~mm} / 0.6^{\circ}$ & ++ & 135 & 183 \\
8 & 30 & 384 & 0.33 & $0.5 \mathrm{~mm} / 0.5^{\circ}$ & ++ & 129 & $131^{\mathrm{a}}$ \\
9 & N/A & N/A & N/A & $2.0 \mathrm{~mm} / 0.6^{\circ}$ & ++ & 129 & 152 \\
10 & 15 & 512 & 0.24 & $0.6 \mathrm{~mm} / 0.4^{\circ}$ & ++ & 100 & 150 \\
11 & 160 & 320 & 0.22 & $0.5 \mathrm{~mm} / 0.3^{\circ}$ & $(+)$ & 104 & $99^{\mathrm{a}}$ \\
\hline
\end{tabular}

${ }^{\mathrm{a}}$ Glucose levels after additional routine imaging, that lasted approx. $20 \mathrm{~min}$ for successful DGE imaging at $3 \mathrm{~T}$, and prolonged infusion times have been suggested [13]. To reduce sensations at the site of injection and to reduce the risk of a potential harmful thrombophlebitis we used an injection protocol that used (1) a reduced concentration of D-glucose (D20) in comparison to previous reports $[5,13,18]$ and (2) a prolonged infusion time of 2-2.5 min in comparison to previous reports at $3 \mathrm{~T}$ and $7 \mathrm{~T}[5,13,18]$. We could minimize motion by this approach, although in some patients motion $>0.5 \mathrm{~mm}$ or $>0.5^{\circ}$ was detectable with concurrent signal changes even after motion correction and dynamic B0 correction.

Although our clinical results in patients with glioma at $3 \mathrm{~T}$ clearly demonstrate a strong association between DGE $\rho$ signal and blood-brain barrier disruption, further validation and evaluation are required to get a better understanding of the underlying physiological sources of signal formation in vivo. A combined effect of reduced $\mathrm{pH}$ within the extracellular space in high-grade gliomas and an increased concentration of D-glucose within the extracellular space when the blood-brain barrier is broken, could explain the findings. As the intracellular glucose concentration and its phosphorylated analogues are not detectable, due to the fast tumor metabolism, they should not contribute significantly to the signal formation [2]. For future measurements at $3 \mathrm{~T}$, higher SNR, reduced motion (e.g. by using inflatable positioning pads) and additional measurement of $\mathrm{pH}$ changes, e.g. based on amine-weighted CEST imaging [19, 20] or 31-P spectroscopy [16], within the tumor and adjacent regions would be helpful to disentangle the influence of different physiologic parameters and artificial signal changes. A further increase in SNR and spatial resolution might be possible with the use of echo planar imaging (EPI) based sequences $[21,22]$, at the cost of potential signal loss in high-grade gliomas with hemorrhage. A whole-brain sequence using spatially non-selective pulses would also remove artifacts due to intensity differences in the slab profile and would allow improved motion correction of the brain. Nonetheless, our first clinical results associating DGE $\rho$ signal with Gd CE promisingly indicate that glucoCEST corresponds more to the disruptions of the blood-brain barrier with Gd uptake than to the molecular tumor profile or tumor grading.

For clinical protocols Gadolinum based imaging currently will remain the gold standard to identify $\mathrm{BBB}$ breakdown. To include DGE $\rho$ imaging within clinical protocols, shorter measurement times and higher SNR would be necessary. But it could be a potential method to avoid or reduce Gadolinium exposition in vulnerable patients in the future, if the association proofs to be strong.

Supplementary Information The online version contains supplementary material available at https://doi.org/10.1007/s10334-021-00982-5.

Acknowledgements The financial support of the Max Planck Society, German Research Foundation (DFG; Grant ZA 814/2-1, support to K. H.), and European Union's Horizon 2020 research and innovation program (Grant Agreement no. 667510, support to A. D., M. K., M. Z., X. G.) is gratefully acknowledged. X. G. is supported by the National Institute for Health Research University College London Hospitals Biomedical Research Centre.

Author contribution $\mathrm{BB}$ - conceptualization; investigation, formal analysis, writing —original draft. $\mathrm{KH}$ - investigation; methodology; software; formal analysis; visualization; writing - review and editing. AD-investigation; methodology; software; writing — review and editing. VR-investigation; writing - review and editing. GT-data curation; resources; writing-review and editing. JS—data curation; resources; writing — review and editing. MS—data curation; resources; writing - review and editing. KS—supervision; resources; methodology; writing — review and editing. UE-supervision; resources; writing-review and editing. $\mathrm{MK}$ - conceptualization; methodology; writing—review and editing. XG—conceptualization; methodology; supervision; writing - review and editing. MZ—conceptualization; methodology; software; writing —original draft. TL—conceptualization; investigation, project administration, writing—original draft. 
Funding Open Access funding enabled and organized by Projekt DEAL.

\section{Declarations}

Conflict of interest BB is co-founder and share-holder of AIRAmed and has received consultancy fees from Medtronic (all outside of the submitted work); G.T. reports personal fees (advisory board, speaker`s fees) from AbbVie, Bayer, Bristol-Myers-Squibb, Medac, Novocure, travel grants from Bristol-Myers-Squibb, educational and travel grants from Novocure, research grants from Roche Diagnostics, research and travel grants from Medac; TL is co-founder, CEO and share-holder of AIRAmed, he has received travel grants from Bayer and presentation fees from Roche (all outside of the submitted work); XG is co-founder, CEO and share-holder of Gold Standard Phantoms; all other authors report no conflicts of interest.

Ethics approval This study was performed in line with the principles of the Declaration of Helsinki. Approval was granted by the Ethics Committee of the University of Tübingen (No. 374/2016BO1).

Informed consent Written informed consent was obtained from all individual participants included in the study.

Open Access This article is licensed under a Creative Commons Attribution 4.0 International License, which permits use, sharing, adaptation, distribution and reproduction in any medium or format, as long as you give appropriate credit to the original author(s) and the source, provide a link to the Creative Commons licence, and indicate if changes were made. The images or other third party material in this article are included in the article's Creative Commons licence, unless indicated otherwise in a credit line to the material. If material is not included in the article's Creative Commons licence and your intended use is not permitted by statutory regulation or exceeds the permitted use, you will need to obtain permission directly from the copyright holder. To view a copy of this licence, visit http://creativecommons.org/licenses/by/4.0/.

\section{References}

1. Walker-Samuel S, Ramasawmy R, Torrealdea F, Rega M, Rajkumar V, Johnson SP, Richardson S, Goncalves M, Parkes HG, Arstad E, Thomas DL, Pedley RB, Lythgoe MF, Golay X (2013) In vivo imaging of glucose uptake and metabolism in tumors. Nat Med 19(8):1067-1072

2. Chan KW, McMahon MT, Kato Y, Liu G, Bulte JW, Bhujwalla ZM, Artemov D, van Zijl PC (2012) Natural D-glucose as a biodegradable MRI contrast agent for detecting cancer. Magn Reson Med 68(6):1764-1773

3. Xu X, Chan KW, Knutsson L, Artemov D, Xu J, Liu G, Kato Y, Lal B, Laterra J, McMahon MT, van Zijl PC (2015) Dynamic glucose enhanced (DGE) MRI for combined imaging of blood-brain barrier break down and increased blood volume in brain cancer. Magn Reson Med 74(6):1556-1563

4. Xu X, Yadav NN, Knutsson L, Hua J, Kalyani R, Hall E, Laterra J, Blakeley J, Strowd R, Pomper M, Barker P, Chan K, Liu G, McMahon MT, Stevens RD, van Zijl PC (2015) Dynamic glucoseenhanced (DGE) MRI: translation to human scanning and first results in glioma patients. Tomogr J Imaging Res 1(2):105-114

5. Paech D, Schuenke P, Koehler C, Windschuh J, Mundiyanapurath S, Bickelhaupt S, Bonekamp D, Bäumer P, Bachert P, Ladd ME, Bendszus M, Wick W, Unterberg A, Schlemmer HP, Zaiss M,
Radbruch A (2017) T1 $\rho$-weighted dynamic glucose-enhanced MR imaging in the human brain. Radiology 285(3):914-922

6. Boyd PS, Breitling J, Zimmermann F, Korzowski A, Zaiss M, Schuenke P, Weinfurtner N, Schlemmer HP, Ladd ME, Bachert P, Paech D, Goerke S (2020) Dynamic glucose-enhanced (DGE) MRI in the human brain at $7 \mathrm{~T}$ with reduced motion-induced artifacts based on quantitative R(1 $\rho)$ mapping. Magn Reson Med 84(1):182-191

7. Schuenke P, Koehler C, Korzowski A, Windschuh J, Bachert P, Ladd ME, Mundiyanapurath S, Paech D, Bickelhaupt S, Bonekamp D, Schlemmer HP, Radbruch A, Zaiss M (2016) Adiabatically prepared spin-lock approach for T1rho-based dynamic glucose enhanced MRI at ultrahigh fields. Magn Reson Med. https://doi.org/10.1002/mrm.26370

8. Schuenke P, Paech D, Koehler C, Windschuh J, Bachert P, Ladd ME, Schlemmer HP, Radbruch A, Zaiss M (2017) Fast and quantitative T1 $\rho$-weighted dynamic glucose enhanced MRI. Sci Rep 7:42093

9. Herz K, Gandhi C, Schuppert M, Deshmane A, Scheffler K, Zaiss M (2019) CEST imaging at $9.4 \mathrm{~T}$ using adjusted adiabatic spinlock pulses for on- and off-resonant T1 $\mathrm{p}$-dominated Z-spectrum acquisition. Magn Reson Med 81(1):275-290

10. Zaiss M, Anemone A, Goerke S, Longo DL, Herz K, Pohmann R, Aime S, Rivlin M, Navon G, Golay X, Scheffler K (2019) Quantification of hydroxyl exchange of D-Glucose at physiological conditions for optimization of glucoCEST MRI at 3, 7 and 9.4 Tesla. NMR Biomed 32(9):e4113

11. Zaiss M, Herz K, Deshmane A, Kim M, Golay X, Lindig T, Bender B, Ernemann U, Scheffler K (2019) Possible artifacts in dynamic CEST MRI due to motion and field alterations. J Magn Reson 298:16-22

12. Herz K, Lindig T, Deshmane A, Schittenhelm J, Skardelly M, Bender B, Ernemann U, Scheffler K, Zaiss M (2019) T1 $\rho$-based dynamic glucose-enhanced (DGE $\rho$ ) MRI at 3 T: method development and early clinical experience in the human brain. Magn Reson Med 82(5):1832-1847

13. Xu X, Sehgal AA, Yadav NN, Laterra J, Blair L, Blakeley J, Seidemo A, Coughlin JM, Pomper MG, Knutsson L, van Zijl PCM (2020) d-glucose weighted chemical exchange saturation transfer (glucoCEST)-based dynamic glucose enhanced (DGE) MRI at 3T: early experience in healthy volunteers and brain tumor patients. Magn Reson Med 84(1):247-262

14. Klein S, Staring M, Murphy K, Viergever MA, Pluim JP (2010) elastix: a toolbox for intensity-based medical image registration. IEEE Trans Med Imaging 29(1):196-205

15. Windschuh J, Zaiss M, Ehses P, Lee JS, Jerschow A, Regatte RR (2019) Assessment of frequency drift on CEST MRI and dynamic correction: application to gagCEST at $7 \mathrm{~T}$. Magn Reson Med 81(1):573-582

16. Mirkes C, Shajan G, Chadzynski G, Buckenmaier K, Bender B, Scheffler K (2016) (31)P CSI of the human brain in healthy subjects and tumor patients at $9.4 \mathrm{~T}$ with a three-layered multi-nuclear coil: initial results. Magma (New York, NY) 29(3):579-589

17. Korzowski A, Weinfurtner N, Mueller S, Breitling J, Goerke S, Schlemmer H-P, Ladd ME, Paech D, Bachert P (2020) Volumetric mapping of intra- and extracellular $\mathrm{pH}$ in the human brain using 31P MRSI at 7T. Magn Reson Med 84(4):1707-1723

18. Knutsson L, Seidemo A, Rydhög Scherman A, Markenroth Bloch K, Kalyani RR, Andersen M, Sundgren PC, Wirestam R, Helms G, van Zijl PCM, Xu X (2018) Arterial input functions and tissue response curves in dynamic glucose-enhanced (DGE) imaging: comparison between glucoCEST and blood glucose sampling in humans. Tomogr J Imaging Res 4(4):164-171

19. Harris RJ, Cloughesy TF, Liau LM, Nghiemphu PL, Lai A, Pope WB, Ellingson BM (2016) Simulation, phantom validation, and clinical evaluation of fast $\mathrm{pH}$-weighted molecular imaging using 
amine chemical exchange saturation transfer echo planar imaging (CEST-EPI) in glioma at $3 \mathrm{~T}$. NMR Biomed 29(11):1563-1576

20. Zaiss M, Xu J, Goerke S, Khan IS, Singer RJ, Gore JC, Gochberg DF, Bachert P (2014) Inverse Z-spectrum analysis for spillover-, MT-, and T1 -corrected steady-state pulsed CEST-MRIapplication to $\mathrm{pH}$-weighted MRI of acute stroke. NMR Biomed 27(3):240-252

21. Mueller S, Stirnberg R, Akbey S, Ehses P, Scheffler K, Stöcker T, Zaiss M (2020) Whole brain snapshot CEST at 3T using 3D-EPI: aiming for speed, volume, and homogeneity. Magn Reson Med 84(5):2469-2483
22. Akbey S, Ehses P, Stirnberg R, Zaiss M, Stöcker T (2019) Wholebrain snapshot CEST imaging at $7 \mathrm{~T}$ using 3D-EPI. Magn Reson Med 82(5):1741-1752

Publisher's Note Springer Nature remains neutral with regard to jurisdictional claims in published maps and institutional affiliations. 